\title{
Un régulateur global de transcription impliqué dans l'association retard mental et $\alpha$-thalassémie (syndrome ATR-X)
}

Les recherches récentes ont insisté sur la fréquence et la variété des syndromes de retard mental liés au chromosome $X: 1,8$ à 3,4 pour 1000 naissances suivant les séries [1]. Un des plus étudiés, sans doute, est celui qui associe ce retard mental à un phénotype d' $\alpha$-thalassémie. Les travaux du groupe de Weatherall à Oxford (GB) avaient en 1990 permis de différencier deux syndromes d' $\alpha$-thalassémic avec retard mental [2,3]. L'un était dû à des grandes délétions télomériques du bras court du chromosome 16 (ATR-16) (m/s $n^{\circ} 1$, vol. 10, $p$. 108). Le syndrome lié à l'X (ATR-X), remarquablement uniforme dans sa présentation, se caractérisait par une déficience mentale beaucoup plus profonde $(\mathrm{QI}<50)$, un pourcentage modéré d'hémoglobine $\mathrm{H}(\mathrm{HbH})$, mais aussi des dysmorphies faciales importantes avec microcéphalie, des anomalies génitales constantes et, de façon variable, d'autres anomalies génétiques. Mais surtout la région des gènes $\alpha$-globine sur le chromosome 16 s'avérait normale. L'atteinte simultanée de deux gènes $\alpha$, par ailleurs présents, suggérait l'action d'un facteur agissant en trans, et une série d'études familiales permettait de situer le locus responsable sur le chromosome X $\left(\mathrm{m} / \mathrm{s} n^{\circ} 1\right.$, vol. 8 , p. 73). A partir des familles alors disponibles, deux séries de données ont dans un premier temps fait progresser les connaissances [4]. On a pu identifier des mères transmettrices. Chez certaines d'entre elles, une recherche fine pouvait mettre en évidence un petit nombre d'inclusions d'HbH dans les globules rouges. Le trait le plus caractéristique était cependant le biais important d'inactivation du chromosome X, étudié par des marqueurs informatifs dont le te kinase ( $P(; K l)$, et cela dans tous les tissus, d'origine mésodermique (cellules sanguines), endodermique (muqueuse buccale) ou ectodermique (bulbes pileux) : contrairement à la situation normale où l'inactivation d'un chromosome $\mathrm{X}$ sur deux est aléatoire dans chaque cellule, un $\mathrm{X}$ semble ici préférenticllement inactivé. L'importance du phénomène était donnée comme indicateur du caractère de transmettrice. Joint au très large spectre des tissus et des systèmes affectés dans la maladie, ce biais d'inactivation de l'X évoquait un gène susceptible d'effets pléiotropes au cours du développement. L'uniformité du syndrome, par ailleurs, était en faveur d'un gène unique contrôlant d'autres gènes. Une autre direction de recherche était une analyse de liaison classique avec des marqueurs connus du chromosome X. Ce travail avait permis de localiser le gène recherché sur un intervalle d'environ $11 \mathrm{cM}$ entre les loci DXS106 et DXYSIX (Xq12q21.31) [4].

Dans un article récent, la même équipe a réussi à localiser le gène en question [5]. Neuf nouvelles familles ont été adjointes à celles qui étaient déjà connues. A partir de ces seize familles, les analyses de liaison ont permis de limiter la zone d'intérêt à $1,4 \mathrm{cM}$ dans la région Xq13.1-q21.1, entre deux marqueurs définis, avec un lod score de 9,56. Cette région du chromosome $\mathrm{X}$ a été très étudiée, et un certain nombre d'ADNc ou de fragments d'ADNc y ont été caractérisés [6].

Une étude moléculaire de la région suspecte a été entreprise chez vingtsix garçons affectés et non apparentés. En utilisant des marqueurs connus, aucun remaniement génomique n'a pu être mis en évidence ; mais l'utilisation de fragments d'ADNc, de 84 pb (E4) ou de plus grande taille $(1000 \mathrm{pb})$ mais de la même région (XNP3/4), notait l'absence d'hybridation chez l'un des vingt-six sujets. L'étude de lymphoblastes normaux immortalisés par le virus d'Epstein-Barr permettait, avec les mêmes sondes, d'identifier par Northern blot un transcrit d'ARNm de 9,5 kb. L'exploration ultérieure de diverses banques d'ADNc de cerveau foetal et de cour humain démontrait enfin que les séquences concernées correspondaient à un gène largement exprimé, supposé être une hélicase et connu sous le nom de XNP [7] ou XH2 [8]. Ces résultats laissaient supposer que le gène $X H 2$ était, au moins partiellement, délété chez un des malades. Une délétion d'environ 2 kb (1973 pb) a pu être confirmée sur des chromosomes $\mathrm{X}$ isolés par cytométrie de flux ; on a aussi montré sur les lymphoblastes immortalisés du malade que cette délétion inhibe la transcription du gène en question.

Le rôle du gène $\mathrm{XH} 2$ semblant démontré chez un sujet, ce même gène a ensuite été exploré chez vingtquatre des malades chez lesquels aucun réarrangement n'avait été retrouvé. Sur des lymphoblastes, on a retrouvé plusieurs cas dans lesquels l'ARNm était diminué ou instable. Une deuxième étape a été l'électrophorèse de fragments d'ADN dénaturé (SSCP pour single strand conformation polymorphism): des anomalies de migration ont été retrouvées chez onze sujets issus de neuf familles. La dernière étape, amplification et séquence, a permis d'identifier neuf mutations différentes. Ces mutations, surtout des mutations faux sens, se groupent dans des régions du gène conservées pendant l'évolution. Le 
fait que ces mutations ne touchent pas des acides aminés essentiels laisse supposer un caractère létal potentiel. Ce remarquable travail expérimental suggère quelques réflexions. On est frappé de la traduction clinique univoque de mutations toutes différentes. On peut supposer que ce gène largement exprimé joue un rôle important dans le développement du système nerveux central, et sans doute aussi dans celui du système urogénital.

Des travaux antérieurs ont montré que le gène $\mathrm{XH} 2$ est soumis à inactivation et n'est donc transcrit qu'à partir du chromosome $\mathrm{X}$ actif. Le biais d'inactivation observé chez les transmettrices serait dû à une sélection somatique négative, c'est-à-dire au développement préférentiel des cellules dont l'X actif porte le gène non muté, moins vraisemblablement à une action directe du centre d'inactivation XIST voisin ; le fait que la sélection soit retrouvée dans tous les tissus laisse supposer une expression embryonnaire de $\mathrm{XH} 2$ précoce, antérieure à la différenciation de l'endoderme.

L'analyse de séquence rapproche $\mathrm{XH} 2$ d'une superfamille très conservée d'hélicases qui présentent une grande variété de fonctions cellulaires [9]. Aucun domaine n'a cependant pu être relié directement à une fonction spécifique, et le meilleur guide reste sans doute l'observation des conséquences phénotypiques des mutations. Dans le cas présent, et par opposition à d'autres syndromes, on ne retrouve pas d'évolution vers la malignité. L'association retrouvée de façon constante avec une $\alpha$-thalassémie fait supposer l'implication de XH2 dans la régulation d'expression des gènes ; plus qu'à une inhibition de telle ou telle étape, ce qu'on observe ressemblerait à une désorganisation globale de la transcription, peut-être par interaction avec des facteurs spécifiques. Pourquoi certains gènes et pas d'autres, les gènes $\alpha$-globine et pas les gènes $\beta$-globine? Les facteurs d'activation, ubiquitaires ou spécifiques, sont les mêmes dans les deux familles de gènes, mais la configuration chromatinienne est très différente [10] ; cela peut laisser supposer une interaction de $\mathrm{XH} 2$ avec la chromatine, nécessaire pour une régulation correcte des gènes $\alpha$-globine et non des gènes $\beta$-globine.

Un dernier point, enfin, est la concentration de maladies génétiques sur cette zone limitée du chromosome X. L'étude dissociée des différents phénotypes devrait être très informative quant à la fonction des diff érents gènes.

D.L.

1. Neri G, Chiurazzi P, Arena JF, Lubs HA. XLMR genes: update 1994. Am J Hum Genet 1994; 51 $542-9$

2. Wilkie AOM, Buckle VJ, Harris PC, Lamb J, Barton NJ, Reeders ST, Lindenbaum RH, Nicholls RD, Barrow M, Bethlenfalvay NC, Hutz $\mathrm{MH}$, Tolmie JL, Weatherall DJ, Higgs DR. Clinical features and molecular analysis of the $\alpha$ thalasse$\mathrm{mia} /$ mental retardation syndromes. I. Cases due to deletions involving chromosome band 16p13.3. Am J Hum Genet 1990 ; 46 : 1112-26.

3. Wilkie AOM, Zeitlin $\mathrm{HC}$, Lindenbaum $\mathrm{RH}$ Buckle VJ, Fischel-Ghodsian N, Chui DHK, Gardner-Medwin D, MacGillivray $\mathrm{MH}$, Weatherall DJ, Higgs DR. Clinical features and molecular analysis of the $\alpha$ thalassemia/mental retardation syndromes. Il. Cases without detectable abnormality of the $\alpha$ globin complex. Am J Hum Genet 1990 ; $46: 1127-40$

4. Gibbons RJ, Suthers GK, Wilkie AOM, Buckle VJ, Higgs DR. X-linked $\alpha$-thalassemia/mental retardation (ATR-X) syndrome: localization to $\mathrm{Xq12-q} 21.31$ by $\mathrm{X}$ inactivation and linkage analysis. Am J Hum Genet 1992 ; 51 : 1136-49.

5. Gibbons RJ, Picketts DJ, Villard L, Higgs DR. Mutations in a putative global transcriptional regulator cause $X$-linked mental retardation with $\alpha$-thalassemia (ATR-X syndrome). Cell 1995; 80 : 837-45.

6. Gecz J, Villard L, Lossi AM, Millasseau P, Djabali $\mathrm{M}$, Fontes $\mathrm{M}$. Physical and transcriptional mapping of $D X S 56-P^{\prime}(; K 11 \mathrm{Mb}$ region: identification of three new transcripts. Hum Mol Genet 1993; $2: 1389-96$

7. Gecz. J, Pollard H, Consalez. G, Villard L, Stayton C, Millasseau P, Khrestchatisky M, Fontes M. Cloning and expression of the murine homologue of a putative human X-linked nuclear protein gene closely linked to $P(; K 1$ in $\mathrm{Xq} 13.3$. Hum Mol Genet $1994 ; 3$ : 39-44.

8. Stayton CL, Dabovic B, Gulisano M, Gecz. J, Broccoli V, Giovanazzi S, Bossolasco M, Monaco L, Rastan S, Boncinelli E, Bianchi ME, Consalez. GG. Cloning and characterization of a new human Xq13 gene, encoding a putative helicase. Hum Mol Genel 1994; 3 : 1957-64.

9. Kolstø $\mathrm{AB}$, Bork $\mathrm{P}$, Kvaløy $\mathrm{K}$, Lindback $\mathrm{T}$, Grønstadt A, Kristensen T, Sander C. Prokaryotic members of a new family of putative helicases with similarity to transcription activator SNF2. I Mol Biol 1993; 230 : 684-8.

10. Labie D. Les mécanismes de contrôle de l'expression des gènes $\alpha$ et $\beta$ globine ne sont pas identiques. méderine/sriences 199 ; 8: 848-51.

\section{BRÈVES}

Lithium et aquaporine-2. Les sels de lithium sont largement utilisés dans le traitement des psychoses maniaco-dépressives; leur effet indésirable le plus fréquent est la polyurie, due à un diabète insipide néphrogénique (DIN), par résistance des cellules du canal collecteur rénal à l'action de la vasopressine (AVP). Un groupe de chercheurs danois [1] a exploré ce phénomène chez le rat. Chez cet animal, le traitement chronique par le lithium entraîne un DIN. L'expression du gène codant pour l'aquaporine-2 (AQP2), le canal de l'eau de la portion médullaire du canal collecteur (voir $\mathrm{m} / \mathrm{s} \mathrm{n}^{\circ} 2$, vol. 11 , p. 299), est réduite de façon prof onde par l'administration de lithium. Cette diminution d'expression se corrige partiellement à l'arrêt du lithium (comme chez l'homme chez lequel la capacité de concentration de l'urine se corrige lentement). La restriction en eau ou l'administration de dDAVP, un agoniste analogue de l'AVP, augmentent l'osmolalité urinaire et l'expression d'AOP2; en effet, la dDAVP, dépourvue d'effets vasculaires sur les récepteurs V1, peut, de ce fait, être administrée à doses fortes, surmontant le blocage de l'adénylyl cyclase produit par le lithium. Marples et al. [1] ont étudié en immuno-microscopie électronique la distribution de l'AQP2 : à l'état basal, AQP2 est trouvée à la membrane apicale et dans des vésicules intracellulaires. L'AVP stimule la mobilisation des molécules d'AQP2 des vésicules vers la membrane apicale où elles s'insèrent. Ainsi, chez les rats recevant du lithium, la restriction en eau augmente l'expression d'AQP2 dans les vésicules intracytoplasmiques alors que la dDAVP concentre l'AQP2 au pôle tout apical des cellules de canal collecteur.

[1. Marples D, et al. J Clin Invest $1995 ; 95$ : 1838-45.] 